Research Article

\title{
Fuzzy Modeling for the Dynamics of Alcohol-Related Health Risks with Changing Behaviors via Cultural Beliefs
}

\author{
Maranya M. Mayengo $\mathbb{D}^{1,2}$ Moatlhodi Kgosimore, ${ }^{3}$ and Snehashish Chakraverty $\mathbb{D}^{4}$ \\ ${ }^{1}$ Nelson Mandela African Institution of Science and Technology (NM-AIST), Arusha, Tanzania \\ ${ }^{2}$ College of Natural and Mathematical Sciences (CNMS), The University of Dodoma, Tanzania \\ ${ }^{3}$ Botswana University of Agriculture and Natural Resources, Botswana \\ ${ }^{4}$ National Institute of Technology (NIT), Rourkela, India \\ Correspondence should be addressed to Maranya M. Mayengo; maranyam@nm-aist.ac.tz
}

Received 18 February 2020; Accepted 26 May 2020; Published 13 July 2020

Academic Editor: Chongxin Liu

Copyright (c) 2020 Maranya M. Mayengo et al. This is an open access article distributed under the Creative Commons Attribution License, which permits unrestricted use, distribution, and reproduction in any medium, provided the original work is properly cited.

\begin{abstract}
In this paper, we propose and analyze a fuzzy model for the health risk challenges associated with alcoholism. The fuzziness gets into the system by assuming uncertainty condition in the measure of influence of the risky individual and the additional death rate. Specifically, the fuzzy numbers are defined functions of the degree of peer influence of a susceptible individual into drinking behavior. The fuzzy basic risk reproduction number $\mathscr{R}_{0}^{f}$ is computed by means of Next-Generation Matrix and analyzed. The analysis of $\mathscr{R}_{0}^{f}$ reveals that health risk associated with alcoholism can be effectively controlled by raising the resistance of susceptible individuals and consequently reducing their chances of initiation of drinking behavior. When perceived respectable individuals in the communities are involved in health education campaign, the public awareness about prevailing risks increases rapidly. Consequently, a large population proportion will gain protection from initiation of drinks which would accelerate their health condition into more risky states. In a situation where peer influence is low, the health risks are likely to be reduced by natural factors that provide virtual protection from alcoholism. However, when the perceived most influential people in the community engage in alcoholism behavior, it implies an increase in the force of influence, and as such, the system will be endemic.
\end{abstract}

\section{Introduction}

Alcohol consumption that leads to health-related epidemics has been one of the leading causes of mortality of individuals worldwide. In Tanzania, just like in other developing countries, the situation gets worse as alcohol consumption becomes an important part of community social activities. However, available literature cite alcohol consumption behavior is one among the risk factors for various health challenges in Tanzanian population [1-9]. While communities identify themselves with alcohol consumption, a large part of the religious population forbid its uptake. Being religious therefore plays an important role in promoting health and molding an individual's behaviors [10, 11]. Increased health defect cases such as malnutrition, chronic pancreatitis diseases, liver cirrhosis, and some types of cancer have been found to be associated to alcoholic behavior [12-19]. While in some parts of the world individuals believed that a low level of alcohol consumption prevents thrombosis [20], recent studies challenge the belief. For example, Griswold et al. [13] established that any amount of alcohol consumption leads to health risks.

Mathematical modeling has been very useful in providing solutions to epidemiology-related problems for both autonomous and nonautonomous system models $[12,14,15,21-$ 29]. While autonomous systems literally mean the use of constant controls, a nonautonomous system occurs when a continuous variable control is used in the system as a 
function of time [22]. However, an application of fuzzy set theory in solving dynamical systems has recently been an interesting research area [30-33].

In this paper, we use an application of fuzzy set theory approach to modify the basic SPLMAR model developed in system (1) into a fuzzy model. Two main distinguishing aspects of the model are as follows: social cultural beliefs as an integral part of the society [34-36] and the staged process in which alcoholism behaviors take in the spread of health risks $[12,14,15,24,26,29]$ are considered.

\section{Fuzzy Model Formulation}

This paper presents crisp model (1) with six time-dependent state variables described as follows: susceptible population, $S$ $(t)$-comprising of individuals susceptible to alcohol drinking habits; protected population, $P(t)$-comprising of individuals with strong religious beliefs that provide them with virtual protection from alcohol drinking; and low-risk population, $L$ $(t)$-individuals at low risk who drink alcoholic beverages once in a while. Others include medium/moderate-risk population, $M(t)$-individuals at medium/moderate risk who drink alcoholic beverages regularly; alcoholics, $A(t)$-highrisk drinkers or alcohol addicts who have developed physical or psychological dependence on alcoholic beverages; and recovered population, $R(t)$-former drinkers who have voluntarily quit alcohol drinking due to various reasons. The model considers multirisk levels established under the drinking cultures of Tanzanian population with active religious beliefs by using constant model parameters defined in Table 1

$$
\left\{\begin{array}{l}
\dot{S}=-\left(\mu+\gamma_{1}+\lambda\right) S+\gamma_{2} P+\omega R+(1-\phi) \pi N, \\
\dot{P}=\phi \pi N+\gamma_{1} S-\left(\mu+\gamma_{2}\right) P+\nu L+\tau M+\psi A, \\
\dot{L}=\lambda \rho S-(\nu+\mu+\sigma) L, \\
\dot{M}=\lambda(1-\rho) S+\sigma L-(\tau+\mu+\delta+\xi) M, \\
\dot{A}=\delta M-(\mu+\alpha+\eta+\psi) A, \\
\dot{R}=\xi M+\eta A-(\omega+\mu) R,
\end{array}\right.
$$

with $S(0)>0, P(0)>0, L(0) \geq 0, M(0) \geq 0, A(0) \geq 0, R(0) \geq 0$, and $N=S+P+L+M+A+R$.

The model is developed on the fact that a nonalcoholic drinker acquires alcohol drinking habits through regular social contacts $[12,37]$ at a rate of $\lambda$ defined as

$$
\lambda=c \beta\left(\frac{L+\theta_{1} M+\theta_{2} A}{N}\right) .
$$

We propose a fuzzy model using $x$ as the variable describing the degree of peer influence of a susceptible individual to initiate drinking behavior. The model development is made under the assumption that both $\beta$ and $\alpha$ are the functions of $x$. The physical meaning of parameters used in the model determined the choice of the two parameters. While $\beta$ is attributed to the spread of health risks associated with alcoholism in the community, $\alpha$ translates the consequences of health risks by means of additional death rate. The fuzzy

\begin{tabular}{|c|c|}
\hline Symbol & Descriptions \\
\hline$\pi$ & Per capita recruitment rate \\
\hline$\varphi$ & $\begin{array}{l}\text { The proportion of recruitment joining the protected } \\
\text { population }\end{array}$ \\
\hline$\mu$ & Natural mortality rate \\
\hline$\lambda$ & Force of peer influence to induce drinking \\
\hline$\beta$ & The measure of influence of the risky individuals \\
\hline$c$ & $\begin{array}{l}\text { The contact rate between a susceptible member } \\
\text { and a drinker necessary to convince the susceptible } \\
\text { member to drink }\end{array}$ \\
\hline$\theta_{1}$ & $\begin{array}{l}\text { The chances of becoming an alcoholic after successful } \\
\text { influence of a moderate-risk drinker }\end{array}$ \\
\hline$\theta_{2}$ & $\begin{array}{l}\text { The chances of becoming an alcoholic after successful } \\
\text { influence of a high-risk drinker }\end{array}$ \\
\hline$\rho$ & $\begin{array}{c}\text { The proportion of susceptible individual recruited to } \\
\text { the low-risk drinking population }\end{array}$ \\
\hline$\omega$ & $\begin{array}{c}\text { The rate at which recovered individuals join susceptible } \\
\text { compartment }\end{array}$ \\
\hline$\gamma_{1}$ & $\begin{array}{c}\text { The rate at which susceptible population joins protected } \\
\text { compartment }\end{array}$ \\
\hline$\gamma_{2}$ & Virtual protection wane rate \\
\hline$v$ & $\begin{array}{c}\text { The rate at which low-risk population joins protected } \\
\text { compartment }\end{array}$ \\
\hline$\tau$ & $\begin{array}{l}\text { The rate at which moderate-risk population joins } \\
\text { protected compartment }\end{array}$ \\
\hline$\psi$ & $\begin{array}{c}\text { The rate at which high-risk population joins protected } \\
\text { compartment }\end{array}$ \\
\hline$\sigma$ & Progressive rate from low to moderate risk compartments \\
\hline$\delta$ & $\begin{array}{l}\text { Progressive rate from moderate- to high-risk } \\
\text { compartments }\end{array}$ \\
\hline$\xi$ & Recovery rate for moderate-risk population \\
\hline$\alpha$ & Alcohol-induced fatality rate \\
\hline$\eta$ & Recovery rate for high-risk population \\
\hline
\end{tabular}

TABLE 1: Model parameters and their description.

numbers $\beta=\beta(x)$ and $\alpha=\alpha(x)$ represent the likelihood that a susceptible individual will drink alcohol after prolonged contact with drinking individuals and the additional death rate induced by alcoholism, respectively. A fuzzy number may be defined as a generalization of a real number referring to a connected set of possible values, rather than referring to one single value, where each possible value is weighted between 0 and 1 . Thus, the fuzzy system (3) of differential equations is established

$$
\left\{\begin{array}{l}
\dot{S}=(1-\phi) \pi N-\left(\mu+\gamma_{1}+\lambda(x)\right) S+\gamma_{2} P+\omega R, \\
\dot{P}=\phi \pi N+\gamma_{1} S-\left(\mu+\gamma_{2}\right) P+v L+\tau M+\psi A, \\
\dot{L}=\lambda(x) \rho S-(\nu+\mu+\sigma) L, \\
\dot{M}=\lambda(x)(1-\rho) S+\sigma L-(\tau+\mu+\delta+\xi) M, \\
\dot{A}=\delta M-(\mu+\alpha(x)+\eta+\psi) A, \\
\dot{R}=\xi M+\eta A-(\omega+\mu) R,
\end{array}\right.
$$




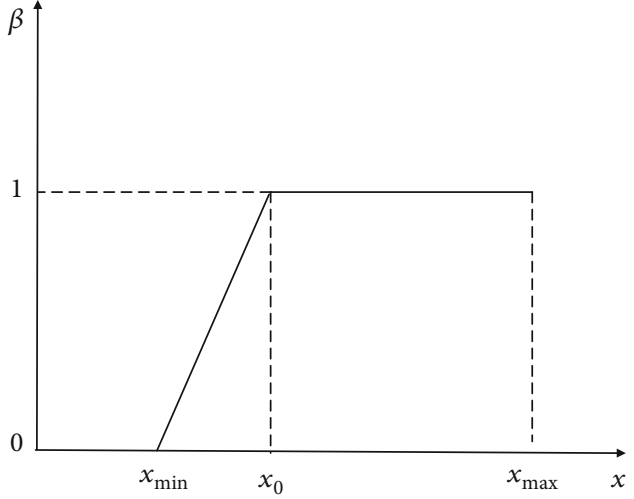

(a)

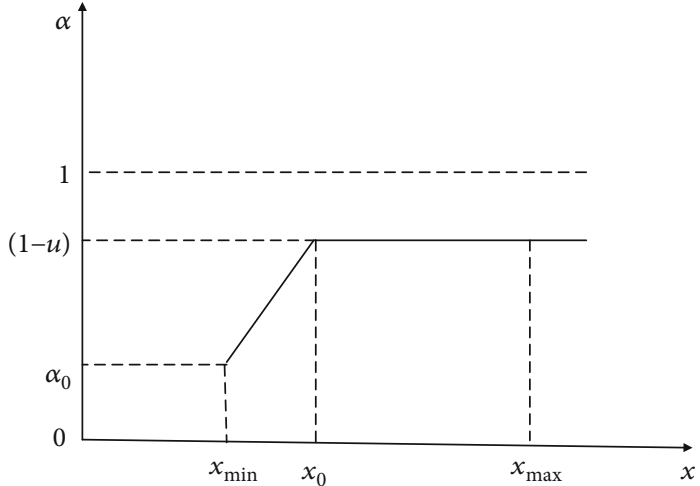

(b)

Figure 1: The graph of membership functions $\beta$ and $\alpha$.

with nonnegative initial conditions of the state variables: $S(0)>0, P(0) \geq 0, L(0) \geq 0, M(0) \geq 0, A(0) \geq 0$ and $R(0) \geq$ 0 where

$$
\begin{aligned}
& N=S+P+L+M+A+R, \\
& \dot{N}=(\pi-\mu) N-\alpha(x) A .
\end{aligned}
$$

It is further assumed that the population is homogeneous and, at the initial level, drinking behavior is acquired by choice. Further, it is assumed that individuals in the recovered population do not develop a permanent immunity system against alcohol drinking. Similarly, individuals in the protected compartment acquire a nonpermanent virtual protection from alcohol drinking for their entire life in the compartment. On the other hand, a susceptible drinker acquires alcohol drinking habits through prolonged social contacts with drinkers in shared drinking venues. The force of peer influence is redefined into

$$
\lambda(x)=c \beta(x)\left(\frac{L+\theta_{1} M+\theta_{2} A}{N}\right) .
$$

\section{Analysis of the Fuzzy System}

Following Verma et al. [38] and Nandi et al. [32], we study the fuzzy SPLMAR model (3) and provide the model analysis and interpretations. The parameters $\beta(x)$ and $\alpha(x)$ can be described through two fuzzy membership functions. For example, considering a situation where a drinking individual interacts with a susceptible member, the minimum amount of the degree of peer influence, $x=x_{\min }$, is required to have an impact on a susceptible member. That is to say, when $x<x_{\min }$, the impact of behavioral influence is considered negligible. The quantity $x_{\min }$ is taken as a parameter whose exact value would depend upon both the attitude, public opinions towards the drinking behavior or the drinking individual, and willingness of a susceptible individual to conform with the peer pressures. As $x$ increases, the behavior inducement rate increases to the maximum which is equal to unity at $x \geq x_{0}$. Further, it is also assumed that the degree of peer influence is bounded above where $x=x_{\max }$ marks an upper bound. Therefore, the values of $x$ with an effect to the system lies in the interval of $x_{\min } \leq x \leq x_{\max }$. The fuzzy membership function for the fuzzy number $\beta(x)$ is given by

$$
\beta(x)=\left\{\begin{array}{l}
0, \quad \text { if } x<x_{\min }, \\
\frac{x-x_{\min }}{x_{0}-x_{\min }}, \quad \text { if } x_{\min } \leq x \leq x_{0}, \\
1, \quad \text { if } x_{0}<x<x_{\max } .
\end{array}\right.
$$

Similarly, we also assume that the addition death rate, $\alpha(x)$, is a fuzzy number as it occurs due to the risk "transmission." When $x<x_{\min }$, we have a negligible amount of risk transmissions implying that the additional death rate, $\alpha(x)$, gets to the minimum level, say $\alpha\left(x<x_{\min }\right)=\alpha_{0}$. As $x$ increases, the additional death rate increases and gets the highest value at $x=x_{0}$. Since the additional death rate may not reach $\alpha(x)=1$ as its highest score due to several limitations, we let the maximum value $\alpha(x)=(1-u)$ for some real number $u$ such that $0<u<\left(1-\alpha_{0}\right)$. We therefore establish the fuzzy membership function of $\alpha(x)$ as follows:

$$
\alpha(x)=\left\{\begin{array}{l}
\alpha_{0}, \quad \text { if } 0 \leq x<x_{\min }, \\
\alpha_{0}+\left(\frac{1-u-\alpha_{0}}{x_{0}-x_{\min }}\right) x, \quad \text { if } x_{\min } \leq x<x_{0}, \\
(1-u), \quad \text { if } x_{0} \leq x \leq x_{\max } .
\end{array}\right.
$$

The graphs of membership functions $\beta(x)$ and $\alpha(x)$ are presented in Figures 1(a) and 1(b), respectively. For the model to be more realistic, we assume that the degree of peer influence of the studied group $\Pi$ differs from among drinking individuals depending on their social influence in the community. Consequently, $\Pi$ can be considered a linguistic variable with varying classifications. If we use $x_{c}$ and $d$ as, respectively, the central value and dispersion of each one of the fuzzy sets assumed by $\Pi$, we model each classification by using a triangular fuzzy number whose membership 


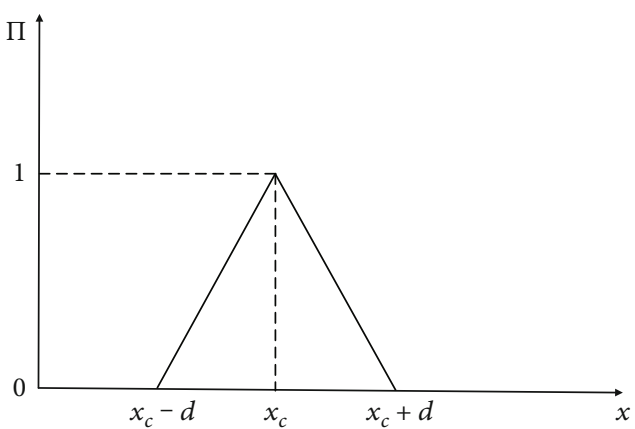

Figure 2: The graph of membership function $\Pi$.

function is given in (8) and the graph of membership function $\Pi$ is presented in Figure 2.

$$
\prod(x)=\left\{\begin{array}{l}
\frac{x-x_{c}+d}{d}, \quad \text { if } x<x_{c}-d, \\
-\frac{x-x_{c}-d}{d}, \quad \text { if } x_{c}-d \leq x \leq x_{c}, \\
0, \quad \text { if } x_{c}+d<x \leq x_{c}+d,
\end{array}\right.
$$

Let $L(x, t), M(x, t)$, and $A(x, t)$ be the family solutions of the fuzzy model system (3). These are the numbers of risky population proportions created as the result of social interactions between the susceptible members and risky individuals with social influence $x$ at time $t$. Now, $L(x, t), M(x, t)$, and $A(x, t)$ are fuzzy numbers which lie in the interval $[0,1]$.

3.1. Existence of Equilibrium Points. The equilibrium points of the fuzzy model (3) can be obtained by setting the RHS to be equal to zero and solve the system simultaneously. Thus, we have

$$
\left\{\begin{array}{l}
0=(1-\phi) \pi N^{*}-\left(\kappa_{1}+\lambda^{*}(x)\right) S^{*}+\gamma_{2} P^{*}+\omega R^{*}, \\
0=\phi \pi N^{*}+\gamma_{1} S^{*}-\kappa_{2} P^{*}+\nu L^{*}+\tau M^{*}+\psi A^{*}, \\
0=\lambda^{*}(x) \rho S^{*}-\kappa_{3} L^{*}, \\
0=\lambda^{*}(x)(1-\rho) S^{*}+\sigma L^{*}-\kappa_{4} M^{*}, \\
0=\delta M^{*}-\left(\kappa_{5}+\alpha(x)\right) A^{*}, \\
0=\xi M^{*}+\eta A^{*}-\kappa_{6} R^{*},
\end{array}\right.
$$

where $\kappa_{1}=\left(\mu+\gamma_{1}\right), \kappa_{2}=\left(\mu+\gamma_{2}\right), \kappa_{3}=(\nu+\mu+\sigma), \kappa_{4}=(\tau+$ $\mu+\delta+\xi), \kappa_{5}=(\mu+\eta+\psi)$, and $\kappa_{6}=(\omega+\mu)$ are simplifying parameters.

The risk-free equilibrium (RFE) point $\left(\varepsilon_{0}\right)$ is obtained when the entire population is free from health risks associated with alcoholism, that is, $L=M=A=0$ implying that $\lambda$ $(x)=0$. Solving system (9) by maintaining risky classes at zero, we have

$$
\varepsilon_{0}=\left(S_{0}^{*}, P_{0}^{*}, L_{0}^{*}, M_{0}^{*}, A_{0}^{*}, R_{0}^{*}\right)=\left((1-\kappa) N_{0}^{*}, \kappa N_{0}^{*}, 0,0,0,0\right),
$$

with the positive constant $\kappa=\left(\pi\left(\phi \mu+\gamma_{1}\right)\right) /\left(\mu\left(\mu+\gamma_{1}+\gamma_{2}\right)\right)$.

Similarly, when $L=0, M=0, A=0$, and consequently $\lambda^{*}$ $(x)=0$, the risk endemic equilibrium REE point $\left(\varepsilon_{1}\right)(11)$ is established.

$$
\varepsilon_{1}=\left(S^{*}, P^{*}, L^{*}, M^{*}, A^{*}, R^{*}\right)
$$

where by $P^{*}, L^{*}, M^{*}, A^{*}$, and $R^{*}$ are defined in

$$
\begin{aligned}
P^{*} & =\frac{1}{\gamma_{2}}\left(\left(\kappa_{1}+\left(1-\omega Q_{3}\right) \lambda^{*}(x)\right) S^{*}-(1-\phi) \pi N^{*}\right), \\
L^{*} & =Q_{0} \lambda^{*}(x) S^{*}, \\
M^{*} & =Q_{1} \lambda^{*}(x) S^{*}, \\
A^{*} & =Q_{2} \lambda^{*}(x) S^{*}, \\
R^{*} & =Q_{3} \lambda^{*}(x) S^{*},
\end{aligned}
$$

and $Q_{0}=\rho / \kappa_{3}, Q_{1}=\left(\kappa_{3}-\rho(\nu+\mu)\right) / \kappa_{3} \kappa_{4}, Q_{2}=\left(\left(\kappa_{3}-\rho(\nu+\right.\right.$ $\mu)) \delta) /\left(\kappa_{3} \kappa_{4}\left(\kappa_{5}+\alpha(x)\right)\right)$, and $Q_{3}=\left(\left(\xi\left(\kappa_{5}+\alpha(x)\right)+\eta \delta\right)\left(\kappa_{3}\right.\right.$ $-\rho(\nu+\mu))) /\left(\kappa_{3} \kappa_{4}\left(\kappa_{5}+\alpha(x)\right) \kappa_{6}\right)$ are the simplifying factors. Thus, the Theorem 1 is established.

Theorem 1. System (3) has a risk-free equilibrium $\varepsilon_{0}=$ $\left((1-\kappa) N_{0}^{*}, \kappa N_{0}^{*}, 0,0,0,0\right)$ and a unique risk endemic equilibrium $\varepsilon_{1}=\left(S^{*}, P^{*}, L^{*}, M^{*}, A^{*}, R^{*}\right)$.

3.2. Bifurcation and Fuzzy Basic Risk Reproduction Number. In this section, we first compute the basic health risk reproduction number, denoted as $\mathscr{R}_{0}$ by using the Next-Generation Matrix method [39, 40]. The value of $\mathscr{R}_{0}$ serves as the determinant to indicate whether the health risk epidemic is possible or not. Based on Diekmann et al.'s study [39], the risk transmission model consists of nonnegative initial conditions together with the following system of equations:

$$
\begin{aligned}
\dot{Z}=\mathscr{F}(Z)-\mathscr{V}(Z)=\left[\begin{array}{c}
\lambda(x) \rho S \\
\lambda(x)(1-\rho) S \\
0
\end{array}\right] \\
-\left[\begin{array}{c}
\kappa_{3} L \\
-\sigma L+\kappa_{4} M \\
-\delta M+\left(\kappa_{5}+\alpha(x)\right) A
\end{array}\right],
\end{aligned}
$$

where $\mathscr{V}(Z)=\mathscr{V}^{-}(Z)-\mathscr{V}^{+}(Z), \quad X=\{S, P, R\}^{T} \in \mathbb{R}^{3}, \quad Z=$ $\{L, M, A\}^{T} \in \mathbb{R}^{3}$, and $(\cdot)^{T}$ denotes transpose. 
We then formulate the risk "transmissions" matrix $F$ and risk "transitions" matrix $V$ such that
Thus, by direct computation, we have

$$
F V^{-1}=(1-\kappa)\left[\begin{array}{ccc}
\frac{c \beta(x) \rho}{\kappa_{3}}\left(1+\frac{\theta_{1} \sigma}{\kappa_{4}}+\frac{\theta_{2} \delta \sigma}{\kappa_{4}\left(\kappa_{5}+\alpha(x)\right)}\right) & \frac{c \beta(x) \rho}{\kappa_{4}}\left(\theta_{1}+\frac{\theta_{2} \delta}{\left(\kappa_{5}+\alpha(x)\right)}\right) & \frac{c \beta(x) \theta_{2} \rho}{\left(\kappa_{5}+\alpha(x)\right)} \\
\frac{c \beta(x)(1-\rho)}{\kappa_{3}}\left(1+\frac{\theta_{1} \sigma}{\kappa_{4}}+\frac{\theta_{2} \delta \sigma}{\kappa_{4}\left(\kappa_{5}+\alpha(x)\right)}\right) & \frac{c \beta(x)(1-\rho)}{\kappa_{4}}\left(\theta_{1}+\frac{\theta_{2} \delta}{\left(\kappa_{5}+\alpha(x)\right)}\right) & \frac{c \beta(x) \theta_{2}(1-\rho)}{\left(\kappa_{5}+\alpha(x)\right)} \\
0 & 0
\end{array}\right] .
$$

The basic risk reproduction number, $\mathscr{R}_{0}$, is given by the dominant eigenvalue of matrix $F V^{-1}$ Therefore,

$$
\mathscr{R}_{0}(x)=c \beta(x)\left(\frac{\left(\rho \kappa_{4}\left(\kappa_{5}+\alpha(x)\right)+\left(\theta_{1}\left(\kappa_{5}+\alpha(x)\right)+\theta_{2} \delta\right)\left((1-\rho) \kappa_{3}+\rho \sigma\right)\right)(1-\kappa)}{\kappa_{3} \kappa_{4}\left(\kappa_{5}+\alpha(x)\right)}\right) .
$$

With appropriate choices of $\rho, \phi \leq 1$, Theorem 2 is established

Theorem 2. The risk-free equilibrium $\varepsilon_{0}$ is locally asymptotic stable when $\mathscr{R}_{0}<1$ and unstable otherwise.

Proof. Consider the Jacobian matrix evaluated at the risk free equilibrium point bellow

$$
J\left(\varepsilon_{0}\right)=\left[\begin{array}{cccccc}
J_{11} & J_{12} & J_{13} & J_{14} & J_{15} & J_{16} \\
J_{21} & J_{22} & J_{23} & J_{24} & J_{25} & \phi \pi \\
0 & 0 & J_{33} & J_{34} & J_{35} & 0 \\
0 & 0 & J_{43} & J_{44} & J_{45} & 0 \\
0 & 0 & 0 & \delta & J_{55} & 0 \\
0 & 0 & 0 & \xi & \eta & -k_{6}
\end{array}\right],
$$

where $J_{11}=-\kappa_{1}+(1-\phi) \pi, J_{12}=\gamma_{2}+(1-\phi) \pi, J_{13}=-c \beta(x)$ $(1-\kappa)+(1-\phi) \pi, J_{14}=-c \beta(x) \theta_{1}(1-\kappa)+(1-\phi) \pi, J_{15}=-$ $c \beta(x) \theta_{2}(1-\kappa)+(1-\phi) \pi, J_{16}=\omega+(1-\phi) \pi, J_{21}=\phi \pi+\gamma_{1}$, $J_{22}=\phi \pi-\kappa_{2}, J_{23}=\phi \pi+\nu, J_{24}=\phi \pi+\tau, J_{25}=\phi \pi+\tau, J_{33}=$ $c \beta(x) \rho(1-\kappa)-\kappa_{3}, \quad J_{34}=c \beta(x) \theta_{1} \rho(1-\kappa), \quad J_{35}=c \beta(x) \theta_{1} \rho$ $(1-\kappa), \quad J_{43}=(1-\rho) c \beta(x)(1-\kappa)+\sigma, J_{44}=c \beta(x) \theta_{1}(1-\rho)$ $(1-\kappa)-\kappa_{4}, J_{45}=c \beta(x) \theta_{1}(1-\rho)(1-\kappa)-\kappa_{4}$, and $J_{55}=-\kappa_{5}$ $-\alpha(x)$

Using a trace determinant method, the risk-free equilibrium point $\varepsilon_{0}$ is locally stable provided that $\operatorname{Tr}\left(J\left(\varepsilon_{0}\right)\right)<0$ and $\operatorname{Det}\left(J\left(\varepsilon_{0}\right)\right)>0$ The trace and determinant of $J\left(\varepsilon_{0}\right)$ are, respectively, given by

$$
\begin{gathered}
\operatorname{Tr}\left(J\left(\varepsilon_{0}\right)\right)=(\pi-\alpha(x))+c \beta(x)\left((1-\rho) \theta_{1}+\rho\right)(1-\kappa)-\sum_{i=1}^{6} \kappa_{i}, \\
\operatorname{Det}\left(J\left(\varepsilon_{0}\right)\right)=\left(1-\mathscr{R}_{0}\right)(\pi-\mu)\left(\mu+\gamma_{1}+\gamma_{2}\right) \kappa_{3} \kappa_{4}\left(\kappa_{5}+\alpha(x)\right) \kappa_{6} .
\end{gathered}
$$

Since $\mu<\pi$, it follows that $\varepsilon_{0}$ is asymptotically stable whenever $\mathscr{R}_{0}<1$ and unstable when $\mathscr{R}_{0}>1$ provided that

$$
\frac{(\pi-\alpha(x))+c \beta(x)\left((1-\rho) \theta_{1}+\rho(1-\kappa)\right.}{\sum_{i=1}^{6} \kappa_{i}}<1 .
$$




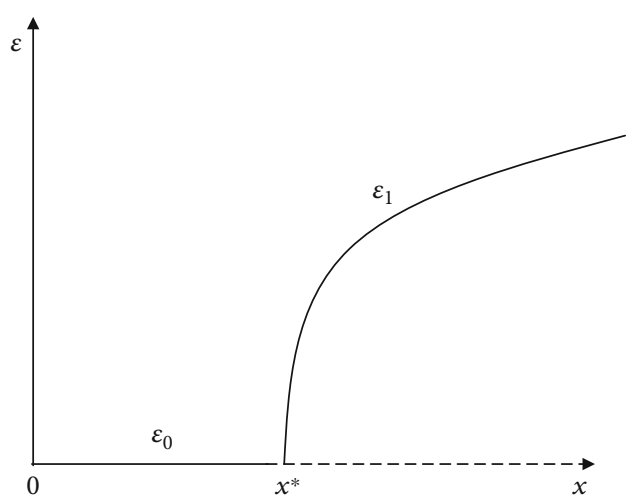

Figure 3: Bifurcation diagram.

The value of $\mathscr{R}_{0}$ may vary significantly depending on different risk dynamics studied in the population or different populations involved in similar studies [33]. Similarly, the stability of the risk-free equilibrium also changes from unstable to stable when $\mathscr{R}_{0}$ increases through 1 . The system acquires a bifurcation at the risk-free equilibrium when $\mathscr{R}_{0}$ $=1$. Suppose that the bifurcation value occurs at $x^{*}$ (see Figure 3) where $x^{*}$ is given as

$$
x^{*}=\frac{a_{4}-\sqrt{a_{2}-2 a_{3}+\left(-1+u+\alpha_{0}\right)^{2}\left(x_{0}-x_{\min }\right)^{2}}}{2 a_{1}\left(-1+u+\alpha_{0}\right)\left(a_{5} \theta_{1}+\rho \kappa_{4}\right)},
$$

provided that $x_{\min } \leq x^{*} \leq x_{0}$ and

$$
\begin{aligned}
a_{1}= & \frac{c(1-\kappa)}{\kappa_{3} \kappa_{4}}, \\
a_{2}= & \left(\left(\left(-1+u+\alpha_{0}\right) x_{\min }+\left(\alpha_{0}+\kappa_{5}\right)\left(x_{\min }-x_{0}\right)\right)\right. \\
& \left.\cdot\left(\theta_{1} a_{5}+\rho \kappa_{4}\right)+\theta_{2} \delta a_{5}\left(x_{\min }+x_{0}\right)\right)^{2} a_{1}^{2}, \\
a_{3}= & \left(x_{0}-x_{\min }\right)\left(-1+u+\alpha_{0}\right)\left(\left(\left(-1+u+\alpha_{0}\right)\right.\right. \\
& \left.+\left(\alpha_{0}+\kappa_{5}\right)\left(x_{0}+x_{\min }\right)\right)\left(\theta_{1} a_{5}+\rho \kappa_{4}\right) \\
& \left.-\left(x_{0}+x_{\min }\right) \theta_{2} \delta a_{5}\right) a_{1}, \\
a_{4}= & \left(\left((u-1) x_{\min }+x_{0} \alpha_{0}\right)\left(\theta_{1} a_{5}+\rho \kappa_{4}\right)\right. \\
& \left.+\left(\left(\theta_{1} a_{5}+\rho \kappa_{4}\right) \kappa_{5}+\theta_{2} \delta a_{5}\right)\left(x_{0}-x_{\min }\right)\right) a_{1} \\
& +\left(-1+u+\alpha_{0}\right)\left(\alpha_{0}-x_{\min }\right), a_{5} \\
= & \left((1-\rho) \kappa_{3}+\rho \sigma\right) .
\end{aligned}
$$

The basic risk reproduction number presented in (21) is the function of $x$, that is $\mathscr{R}_{0}=\mathscr{R}_{0}(x)$, but it can not be referred to as the fuzzy number since it is open to values exceeding unity. It is clear that $\mathscr{R}_{0}(x)$ is the function of the degree of social influence in the spread of the behavior. However, both $\beta(x)$ and $\alpha(x)$ incline to their maximum level whenever $x \geq x_{0}$, and as such, we have

$$
\mathscr{R}_{0}(u)=c\left(\frac{\left(\rho \kappa_{4}\left(\kappa_{5}+(1-u)\right)+\left(\theta_{1}\left(\kappa_{5}+(1-u)\right)+\theta_{2} \delta\right)\left((1-\rho) \kappa_{3}+\rho \sigma\right)\right)(1-\kappa)}{\kappa_{3} \kappa_{4}\left(\kappa_{5}+(1-u)\right)}\right) .
$$

We introduce a positive number $\epsilon_{0}$ such that $\epsilon_{0} \mathscr{R}_{0}(x)$ $\leq 1$, with an appropriate choices of $\epsilon_{0}$; we have a fuzzy set $\epsilon_{0} \mathscr{R}_{0}(x)$ whose fuzzy expected value, $\operatorname{FEV}\left[\epsilon_{0} \mathscr{R}_{0}(x)\right]$, can be well defined. Therefore, the fuzzy basic risk reproduction number $\mathscr{R}_{0}^{f}$, which can be defined as the average number of secondary risk cases caused by one infected node introduced into entirely susceptible nodes [31], is given by

$$
\mathscr{R}_{0}^{f}=\frac{1}{\epsilon_{0}} \operatorname{FEV}\left[\epsilon_{0} \mathscr{R}_{0}(x)\right] .
$$

We know from the definition of fuzzy expected value that

$$
\operatorname{FEV}\left[\epsilon_{0} \mathscr{R}_{0}(x)\right]=\sup _{0 \leq y \leq 1} \inf [y, k(y)]
$$

where $k(y)=\Phi\left\{z \in \Omega: \epsilon_{0} \mathscr{R}_{0}(x) \geq y\right\}=\Phi(\Omega)$ is a fuzzy measure. For the purpose of this study, the possibility measure is given by

$$
\Phi(\Omega)=\sup _{x \in X} \prod(x), \quad \Omega \subset \mathbb{R} .
$$

From $\operatorname{FEV}\left[\epsilon_{0} \mathscr{R}_{0}(x)\right]$, for a monotonic function $\mathscr{R}_{0}(x)$, with set $X$ given as an interval $\left[x^{*}, x_{\max }\right]$, we let $x^{*} \in X$ be the solution of the following equation:

$$
\epsilon_{0} c \beta(x)\left(\frac{\left(\rho \kappa_{4}\left(\kappa_{5}+\alpha(x)\right)+\left(\theta_{1}\left(\kappa_{5}+\alpha(x)\right)+\theta_{2} \delta\right)\left((1-\rho) \kappa_{3}+\rho \sigma\right)\right)(1-\kappa)}{\kappa_{3} \kappa_{4}\left(\kappa_{5}+\alpha(x)\right)}\right)=y .
$$




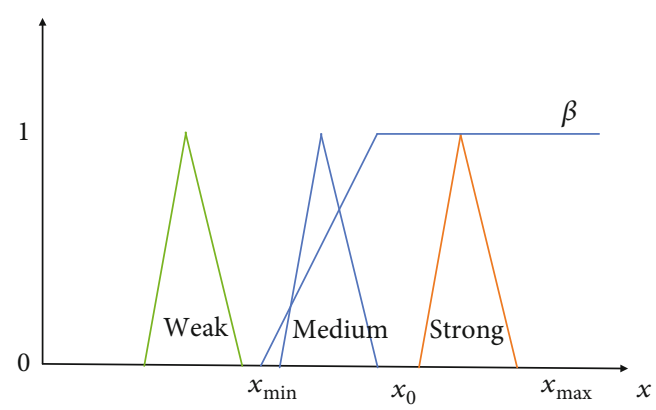

Figure 4: Classification of linguistic variable $\Pi$.

Thus,

$$
k(y)=\Phi\left[x^{*}, x_{\max }\right]=\sup _{x^{*} \leq x \leq x_{\max }} \prod(x)
$$

where $k(0)=1$ and $k(1)=\prod\left(x_{\max }\right)$.

Now, $\operatorname{FEV}\left(\epsilon_{0} \mathscr{R}_{0}\right)$ can be determined by considering the linguistic variable $\Pi$ in three classes: "weak $\Pi_{-}$," "medium $\Pi_{-}^{+}$," and "strong $\Pi^{+}$." Each of these classification is a fuzzy number based on $x_{\min }, x_{0}$, and $x_{\max }$ as shown in Figure 4 . The classification of the degree of social influences in the community can be explained in three different cases as follows:

Case 1. In this case, we consider the weak degree of social influence $\left(\Pi_{-}\right)$where we define that $x_{c}+d<x_{\min }$. Suppose that there exists $\bar{x}$ such that $\mathscr{R}_{0}(\bar{x})=\mathscr{R}_{0}^{f}$; if $x_{c}+d<\bar{x} \mathrm{c}$, we have

$$
k(y)=\sup _{\bar{x} \leq x \leq x_{\max }} \prod(x)=0, \quad \forall y \in[0,1] .
$$

We establish that FEV $\left[\epsilon_{0} \mathscr{R}_{0}\right]=0<\epsilon_{0}$. This can be translated that the fuzzy basic risk reproduction number $\mathscr{R}_{0}^{f}<1$, and hence, the extinction of the health risk is associated with alcoholism.

Case 2. In this case, we consider the medium degree of social influence $\left(\Pi_{-}^{+}\right)$where we define that $x_{c}-d<x_{\min }$ and $x_{c}+$ $d<x_{0}$ giving.

$k(y)=\left\{\begin{array}{l}1, \quad \text { if } 0 \leq y<\epsilon_{0} \mathscr{R}_{0}\left(x_{c}\right), \\ \prod(\bar{x}), \quad \text { if } \epsilon_{0} \mathscr{R}_{0}\left(x_{c}\right) \leq y \leq \epsilon_{0} \mathscr{R}_{0}\left(x_{c}+d\right), \\ 0, \quad \text { if } \epsilon_{0} \mathscr{R}_{0}\left(x_{c}+d\right)<y \leq 1 .\end{array}\right.$

For $d>0, k(y)$ is the continuous function giving $k(0)=1$ and $k(1)=0$. This translates $\mathscr{R}_{0}^{f}$ as the fixed point $k$ and $\mathscr{R}_{0}\left(x_{c}\right)<\mathscr{R}_{0}^{f}<\mathscr{R}_{0}\left(x_{c}+d\right)$. Since $\mathscr{R}_{0}(x)$ is a continuous monotonic (increasing) function, the Intermediate Value Theorem suggests that there exists $\bar{x}$ with $x_{c}<\bar{x}<x_{c}+d$ in which the values of $\mathscr{R}_{0}(\bar{x})$ and $\mathscr{R}_{0}^{f}$ coincide such that $\mathscr{R}_{0}^{f}$ $=\mathscr{R}_{0}(\bar{x})>\mathscr{R}_{0}\left(x_{c}\right)$. Further, the average number of fuzzy basic risk reproduction number $\mathscr{R}_{0}^{f}$ is higher than the num- ber of secondary risk cases $\mathscr{R}_{0}\left(x_{c}\right)$ due to the medium level of social influence implying that the health risk associated with alcoholism is endemic.

Case 3. In this case, we consider the strong degree of social influence $\left(\Pi^{+}\right)$by defining $x_{c}-d>x_{0}$ and $x_{c}+d<x_{\max }$.

The results in (35) can be established.

$$
k(y)=\left\{\begin{array}{l}
1, \quad \text { if } 0 \leq y<\epsilon_{0} \mathscr{R}_{0}\left(x_{c}\right), \\
\prod(\bar{x}), \quad \text { if } \epsilon_{0} \mathscr{R}_{0}\left(x_{c}\right) \leq y<\epsilon_{0} \mathscr{R}_{0}\left(x_{c}+d\right), \\
0, \quad \text { if } \epsilon_{0} \mathscr{R}_{0}\left(x_{c}+d\right) \leq y \leq 1 .
\end{array}\right.
$$

For any given $d>0, k(y)$ is a monotonically decreasing and continuous function with $k(0)=1$ and $k(1)=0$.

Therefore, $\operatorname{FEV}\left[\epsilon_{0} \mathscr{R}_{0}\right]$ is established as a fixed point such that

$$
\epsilon_{0} \mathscr{R}_{0}\left(x_{c}\right)<\operatorname{FEV}\left[\epsilon_{0} \mathscr{R}_{0}\right]<\epsilon_{0} \mathscr{R}_{0}\left(x_{c}+d\right)
$$
results:

By dividing by $\epsilon_{0}$ throughout, we have the following

$$
\mathscr{R}_{0}\left(x_{c}\right)<\mathscr{R}_{0}^{f}<\mathscr{R}_{0}\left(x_{c}+d\right) .
$$

Now, since $\mathscr{R}_{0}^{f}>1$, it translates into endemic health risks associated with alcoholism.

3.3. Risk Control in Fuzzy Epidemic System. In this section, we perform the control analysis of the risk estimation in the population by using the fuzzy basic risk threshold $\mathscr{R}_{0}^{f}=\mathscr{R}_{0}$ $(\bar{x})$. The spread of health risk in the proposed fuzzy SPLMAR model (3) depends on the degree of the social influence $x$ as an input value of the transmission factor $\beta(x)$. The description of existence and stability of the risk in the system is case-wise presented hereunder. Since the proposed fuzzy system (3) represents a family of systems depending on the parameter $x$, these family systems can be simplified by a unique system of equations with the same results. It is shown that the bifurcation occurs at $x=x^{*}$, that is, $\mathscr{R}_{0}\left(x^{*}\right)=1$.

(1) Weak influence: in this case, we have $x<x_{\min }$ giving $\mathscr{R}_{0}=0$ suggesting the extinction of the health risks associated with alcoholism in the community.

(2) Medium influence: in this case, three possibilities may arise as follows:

(i) If $x<x^{*}$, then $\mathscr{R}_{0}<1$ suggesting the risk free community

(ii) If $x=x^{*}$, then $\mathscr{R}_{0}=1$ an indication of risk bifurcation

(iii) If $x>x^{*}$, then $\mathscr{R}_{0}>1$ implying the risk endemic in the community

(3) Strong influence: in this case, we have $x \in\left[x_{0}, x_{\max }\right]$ giving 


$$
\mathscr{R}_{0}(u)=c\left(\frac{\left(\rho \kappa_{4}\left(\kappa_{5}+(1-u)\right)+\left(\theta_{1}\left(\kappa_{5}+(1-u)\right)+\theta_{2} \delta\right)\left((1-\rho) \kappa_{3}+\rho \sigma\right)\right)(1-\kappa)}{\kappa_{3} \kappa_{4}\left(\kappa_{5}+(1-u)\right)}\right) .
$$

The spread of the health risks depends upon the parameter $u$. Let $u^{*}$ be an improved value of $u$; we can establish three possibilities in which the spread of health risks takes as follows:

(i) If $0 \leq u<u^{*}$, then $\mathscr{R}_{0}(u)<1$ suggesting the health risks would be cleared in the community

(ii) If $0 \leq u=u^{*}$, then $\mathscr{R}_{0}(u)=1$ implying that the system passes through a bifurcation state

(iii) If $0 \leq u^{*}<u$, then $\mathscr{R}_{0}(u)>1$ suggesting that the health risk problem would spread out in the system

\section{Discussion and Conclusion}

In this work, we propose and analyze the fuzzy SPLMAR model with the aim of using fuzzy set theory in the model system. Although all the parameters associated in the model system are important, in an uncertain environment, we consider the selected two most important parameters $\beta$ and $\alpha$ representing the probability that a susceptible individual will drink alcohol after prolonged contact with drinking individuals and the additional death rate induced by alcoholism, respectively. In particular, the two parameters $\beta$ and $\alpha$ are considered fuzzy numbers and functions of the degree of force of influence, whose membership functions are well defined. Later, the fuzzy basic risk reproduction number $\mathscr{R}_{0}^{f}(x)$ is computed.

Since the basic risk reproduction number $\mathscr{R}_{0}$ is directly related to varied factors of drinking settings, obtained by considering the classical model system (1) with constant parameters $\beta$ and $\alpha$, it implied that the variation of the risky population is always positive. However, when $\beta$ is considered a fuzzy set, it provide us with an additional information regarding the health risk dynamics. The health risks associated with alcoholism can be effectively controlled by controlling the fuzzy basic risk reproduction number $\mathscr{R}_{0}^{f}$. The analysis of the fuzzy model suggests that $\mathscr{R}_{0}^{f}$ can be reduced by increasing the value of $x_{\min }$. This may be done through provision of public health education which increases the resistance of susceptible individuals and prevent them from initiation of drinking behavior which would accelerate their health condition into more risky states. It is generally observed that if the amount of degree of peer of influence to an individual is low, then the alcohol-related health risks in the community may not be the most effective. The reason is natural factors in the community provide virtual immunity from deeply engaging into alcoholic behaviors. However, when the perceived most influential people in the community engage in alcoholism, it implies an increase in the degree of force of influence, and as such, the system will be endemic.

In a future work, some other parameters may be considered with different functionalities under the uncertainty envi- ronment. However, the present model can be applied to those types of diseases or conditions which spread through direct contact between susceptible and infected individuals.

\section{Data Availability}

No data is included.

\section{Conflicts of Interest}

The authors declare that they have no conflicts of interest.

\section{References}

[1] T. T. H. Ao, N. E. Sam, E. J. Masenga, G. R. Seage III, and S. H. Kapiga, "Human immunodeficiency virus type 1 among bar and hotel workers in northern Tanzania: the role of alcohol, sexual behavior, and herpes simplex virus type 2," Sexually Transmitted Diseases, vol. 33, no. 3, pp. 163-169, 2006.

[2] R. Edwards, N. Unwin, F. Mugusi et al., "Hypertension prevalence and care in an urban and rural area of Tanzania," Journal of Hypertension, vol. 18, no. 2, pp. 145-152, 2000.

[3] S. H. Kapiga, N. E. Sam, H. Bang et al., "The role of herpes simplex virus type 2 and other genital infections in the acquisition of hiv-1 among high-risk women in northern Tanzania," The Journal of Infectious Diseases, vol. 195, no. 9, pp. 1260-1269, 2007.

[4] S. H. Kapiga, N. E. Sam, J. F. Shao et al., "Herpes simplex virus type 2 infection among bar and hotel workers in northern Tanzania: prevalence and risk factors," Sexually Transmitted Diseases, vol. 30, no. 3, pp. 187-192, 2003.

[5] S. H. Kapiga, N. E. Sam, J. F. Shao et al., "HIV-1 epidemic among female bar and hotel workers in northern Tanzania: risk factors and opportunities for prevention," JAIDS Journal of Acquired Immune Deficiency Syndromes, vol. 29, no. 4, pp. 409-417, 2002.

[6] G. P. Kilonzo, "Production and consumption of alcohol in Tanzania and alcohol-related accidents," in Maula et al., Alcohol in Developing Countries, pp. 185-192, Nordic Council for Alcohol and Drug Research, Helsinki, 1990.

[7] T. Mitsunaga and U. Larsen, "Prevalence of and risk factors associated with alcohol abuse in Moshi, North-Ern Tanzania," Journal of Biosocial Science, vol. 40, no. 3, pp. 379-399, 2008.

[8] P. Nikander, R. Honkanen, P. Huttunen, G. Kilonzo, and Y. Pitkanen, Role of Alcohol in Injuries Treated in the Muhimbili Medical Centre Casualty Department, Dar Es Salaam, Tanzania, Pombe: Report of Alcohol Research Project in Tanzania, 1988.

[9] Z. Sa and U. Larsen, "Gender inequality and HIV-1 infection among women in Moshi, Tanzania," Journal of Biosocial Science, vol. 39, 2007.

[10] J. M. Francis, H. A. Weiss, G. Mshana, K. Baisley, H. Grosskurth, and S. H. Kapiga, "The epidemiology of alcohol use and alcohol use disorders among young people in northern Tanzania," PLoS One, vol. 10, no. 10, article e0140041, 2015. 
[11] J. M. Wallace Jr. and T. A. Forman, "Religion's role in promoting health and reducing risk among American youth," Health Education \& Behavior, vol. 25, no. 6, pp. 721-741, 2016.

[12] C. P. Bhunu, "A mathematical analysis of alcoholism," World Journal of Modelling and Simulation, vol. 8, no. 2, pp. 124134, 2012.

[13] GBD 2016 Alcohol Collaborators, “Alcohol use and burden for 195 countries and territories, 1990-2016: a systematic analysis for the Global Burden of Disease Study 2016," The Lancet, vol. 392, no. 10152, pp. 1015-1035, 2018.

[14] H. F. Huo and Y. P. Liu, "The analysis of the SIRS alcoholism models with relapse on weighted networks," Springerplus, vol. 5, no. 1, p. 722, 2016.

[15] D. Jernigan, Alcohol Use. Comparative Quantification of Health Risks, World Health Organization, 2004.

[16] C. J. Murray, A. D. Lopez, and WHO, The global burden of disease: a comprehensive assessment of mortality and disability from diseases, injuries, and risk factors in 1990 and projected to 2020: summary, World Health Organization, 1996.

[17] WHO, "The global burden of disease 2004. Technical report," World Health Organization, 2004, https://www.who.int/ healthinfo/global_burden_disease/GBD_report_2004update_ full.pdf.

[18] WHO, Harmful Use of Alcohol. Technical Report, World Health Organization, 2009.

[19] WHO, The Global Status Report on Alcohol and Health 2014. Technical Report, World Health Organization, 2014.

[20] M. Gronbaek, "The positive and negative health effects of alcohol- and the public health implications," Journal of Internal Medicine, vol. 265, no. 4, pp. 407-420, 2009.

[21] I. K. Adu, M. A. R. E. N. Osman, and C. Yang, "Mathematical model of drinking epidemic," British Journal of Mathematics \& Computer Science, vol. 22, no. 5, pp. 1-10, 2017.

[22] H. W. Berhe, O. D. Makinde, and D. M. Theuri, "Optimal control and cost-effectiveness analysis for dysentery epidemic model," Applied Mathematics \& Information Sciences, vol. 12, no. 6, pp. 1183-1195, 2018.

[23] A. Hugo, O. D. Makinde, S. Kumar, and F. F. Chibwana, "Optimal control and cost effectiveness analysis for Newcastle disease eco-epidemiological model in Tanzania," Journal of Biological Dynamics, vol. 11, no. 1, pp. 190-209, 2016.

[24] J. Mushanyu and F. Nyabadza, "A risk-structured model for understanding the spread of drug abuse," International Journal of Applied and Computational Mathematics, vol. 4, no. 2, 2018.

[25] J. Mushanyu, F. Nyabadza, G. Muchatibaya, and A. G. R. Stewart, "Modelling drug abuse epidemics in the presence of limited rehabilitation capacity," Bulletin of Mathematical Biology, vol. 78, no. 12, pp. 2364-2389, 2016.

[26] S. Mushayabasa, "The role of optimal intervention strategies on controlling excessive alcohol drinking and its adverse health effects," Journal of Applied Mathematics, vol. 2015, Article ID 238784, 11 pages, 2015.

[27] C. J. Silva and D. F. M. Torres, "Optimal control for a tuberculosis model with reinfection and post-exposure interventions," Mathematical Biosciences, vol. 244, no. 2, pp. 154-164, 2013.

[28] S. D. Walsh, A. Djalovski, M. Boniel-Nissim, and Y. HarelFisch, "Parental, peer and school experiences as predictors of alcohol drinking among first and second generation immigrant adolescents in Israel," Drug and Alcohol Dependence, vol. 138, pp. 39-47, 2014.
[29] H. Xiang, N. N. Song, and H. F. Huo, "Modelling effects of public health educational campaigns on drinking dynamics," Journal of Biological Dynamics, vol. 10, no. 1, pp. 164-178, 2016.

[30] E. Massad, N. R. S. Ortega, L. C. de Barros, and C. J. Struchiner, Fuzzy Logic in Action: Applications in Epidemiology and Beyond, vol. 232, Springer Science \& Business Media, 2008.

[31] B. K. Mishra and S. K. Pandey, "Fuzzy epidemic model for the transmission of worms in computer network," Nonlinear Analysis: Real World Applications, vol. 11, no. 5, pp. 43354341, 2010.

[32] S. K. Nandi, S. Jana, M. Manadal, and T. K. Kar, "Analysis of a fuzzy epidemic model with saturated treatment and disease transmission," International Journal of Biomathematics, vol. 11, no. 1, article 1850002, 2018.

[33] R. Verma, S. P. Tiwari, and R. K. Upadhyay, "Fuzzy modeling for the spread of influenza virus and its possible control," Computational Ecology and Software, vol. 8, no. 1, p. 32, 2018.

[34] J. R. Haber, J. D. Grant, T. Jacob, L. B. Koenig, and A. Heath, "Alcohol milestones, risk factors, and religion/spirituality in young adult women," Journal of Studies on Alcohol and Drugs, vol. 73, no. 1, pp. 34-43, 2012.

[35] K. S. Kendler, C. O. Gardner, and C. A. Prescott, "Religion, psychopathology, and substance use and abuse; a multimeasure, genetic-epidemiologic study," American Journal of Psychiatry, vol. 154, no. 3, pp. 322-329, 1997.

[36] H. G. Koenig, Handbook of Religion and Mental Health, Academic Press, San Diego, CA, 1998.

[37] F. Sánchez, X. Wang, C. Castillo-Chávez, D. M. Gorman, and P. J. Gruenewald, "Drinking as an epidemic-a simple mathematical model with recovery and relapse," in Therapist's Guide to Evidence-Based Relapse Prevention, pp. 353-368, Elsevier, 2007.

[38] R. Verma, S. P. Tiwari, and R. K. Upadhyay, "Dynamical behaviors of fuzzy sir epidemic model," in Advances in Fuzzy Logic and Technology 2017, pp. 482-492, Springer, 2017.

[39] O. Diekmann, J. A. P. Heesterbeek, and M. G. Roberts, “The construction of next-generation matrices for compartmental epidemic models," Journal of The Royal Society Interface, vol. 7, no. 47, pp. 873-885, 2010.

[40] P. van den Driessche and J. Watmough, "Reproduction numbers and sub-threshold endemic equilibria for compartmental models of disease transmission," Mathematical Biosciences, vol. 180, no. 1-2, pp. 29-48, 2002. 